\title{
Extension of the 1-D characteristic open boundary conditions to the diffuse interface model
}

\author{
J. Desmarais ${ }^{1} \&$ J. Kuerten ${ }^{1,2}$ \\ ${ }^{1}$ Eindhoven University of Technology, The Netherlands \\ ${ }^{2}$ Twente University of Technology, The Netherlands
}

\begin{abstract}
A novel technique is developed for solving multi-phase flows in unbounded domains using the Diffuse Interface Model (DIM) in 1-D. It extends open boundary conditions originally designed for the Navier-Stokes equations. The non-dimensional formulation of the DIM makes it possible to generalize the approach to any fluid. The equations support a steady state whose analytical approximation close to the critical point depends only on temperature. This feature enables the use of detectors at the boundaries switching between conventional boundary conditions in bulk phases and a multi-phase strategy in interfacial regions. The technique is applied to fluids experiencing a phase transition where the interface between the phases travels through one of the boundaries. When the interface crossing the boundary is fully developed, the technique greatly improves results relative to cases where conventional boundary conditions can be used. Limitations appear when the interface crossing the boundary is not a stable equilibrium between the two phases: the terms responsible for creating the true balance between the phases perturb the interior solution. The approach can be extended to multiple spatial dimensions.

Keywords: multi-phase flows, open boundary conditions, diffuse interface model, characteristics, interface-boundary interaction, Navier-Stokes.
\end{abstract}




\section{Introduction}

The Diffuse Interface Model (DIM) offers the attractive feature to accurately describe multi-phase flows with only one set of equations for the entire domain. These equations are very similar to the Navier-Stokes (NS) equations but contain additional terms to account for interfacial forces and energy conversion. However, their numerical integration requires a very fine grid to capture the interface. This grid needs to be truncated to reduce the computational costs. Truncating domains while simulating infinite fields requires special boundary conditions, as waves supported by the governing equations may be reflected by the boundaries and ruin the interior solution.

In computational aerodynamics, the majority of the literature focuses on open boundary conditions for the NS equations. Thompson [1] considered the simplified linearized Euler equations at the edges with the approach developed by Hedstrom [2]. Poinsot and Lele [3] combined the latter with the analysis from Gustafsson and Sundstrom [4] to extend the boundary conditions for viscous flows, and Sutherland and Kennedy [5] proposed a strategy for mixed component reactive flows.

Despite the active research in designing open boundary conditions for the NS equations, the extension to the DIM remains unsolved as two major features prevent a straightforward application. In the simplest example where a fluid experiences phase transition from liquid to vapor, a constant velocity field will cause a moving interface, and the liquid will replace the vapor: the far field boundary conditions change during the simulation. What is more, close to the interface, the non conventional terms are dominant and present elliptic properties that can undermine the previous approach.

This paper proposes boundary conditions for the DIM in one dimension that minimize interface-boundary interactions. The latter modifies the conventional formulation only in the multi-phase region.

In section 2, the DIM governing equations and the approximation of the steady state are presented. Based on these equations, extension of the 1-D characteristics is derived in section 3. Results of applying the proposed boundary conditions to an interface convected by a constant velocity field are discussed in section 4 . Three domains are distinguished for the error analysis: the entire computational domain, $D_{\mathrm{C}}$, subdivided into the region needed to apply the boundary conditions, $D_{\mathrm{B}}$, and the domain of interest, $D_{\mathrm{I}}=D_{\mathrm{C}} \backslash D_{\mathrm{B}}$. Conclusions and further developments are discussed in section 5 .

\section{Implementation of the Diffuse Interface Model}

In this section, the DIM governing equations and the steady state approximation are presented. The integration numerical schemes are then discussed. 


\subsection{Scaling quantities for the non-dimensional formulation}

The formulation presented by Pecenko et al. [6] consists of three conservation equations and the Van der Waals equation of state:

$$
\begin{aligned}
\rho_{t}+\nabla \cdot(\boldsymbol{\rho u}) & =0 \\
(\rho \boldsymbol{u})_{t}+\nabla \cdot(\rho \boldsymbol{u u}) & =\nabla \cdot(\boldsymbol{d}+\boldsymbol{T}) \\
(\rho E)_{t}+\nabla \cdot((\rho E) \boldsymbol{u}) & =\nabla \cdot((\boldsymbol{d}+\boldsymbol{T}) \cdot \boldsymbol{u})-\nabla \cdot \boldsymbol{j}_{\mathrm{F}}-\nabla \cdot \boldsymbol{j}_{\mathrm{E}}
\end{aligned}
$$

where $\rho$ is the mass density, $\boldsymbol{u}$ the velocity and $E$ the specific total energy.

The viscous stress tensor, $\boldsymbol{d}$, is computed under the Newtonian fluid formulation. The conduction flux, $\boldsymbol{j}_{\mathrm{F}}$, is given by Fourier's conduction law. The Korteweg tensor, $\boldsymbol{T}$, and the interstitial work, $\boldsymbol{j}_{\mathrm{E}}$, account respectively for the interstitial forces and work. They are computed using the expressions derived by Anderson and McFadden [7] and Dunn and Serrin [8].

$$
\begin{aligned}
\boldsymbol{d} & =\mu\left(\nabla \boldsymbol{u}+\nabla \boldsymbol{u}^{\top}-\frac{2}{3}(\nabla \cdot \boldsymbol{u}) \boldsymbol{I}\right) \\
\boldsymbol{j}_{\mathrm{F}} & =-\lambda \nabla T \\
\boldsymbol{T} & =\left(-P+\rho K \Delta \rho+\frac{1}{2} K|\nabla \rho|^{2}\right) \boldsymbol{I}-K \nabla \rho \otimes \nabla \rho \\
\boldsymbol{j}_{\mathrm{E}} & =K(\rho \nabla \cdot \boldsymbol{u}) \nabla \rho
\end{aligned}
$$

where $\mu$ is the dynamic viscosity, $\lambda$ the thermal conductivity, $T$ the temperature, $P$ the pressure, $K$ the capillarity constant, and $\boldsymbol{I}$ the identity tensor.

The conventional energy formulation is extended to take into account the modifications induced by the interfacial forces:

$$
\rho E=\frac{1}{2} \rho|\boldsymbol{u}|^{2}+\rho e+\frac{1}{2} K|\nabla \rho|^{2}
$$

where $e$ is the specific internal energy.

The equations of state chosen by Pecenko to close the governing equations are derived from the Van der Waals formulation:

$$
P=\frac{R T \rho}{M-b \rho}-\frac{a}{M^{2}} \rho^{2} \Rightarrow e=c_{V} T-\frac{a}{M^{2}} \rho
$$

where $R$ is the perfect gas constant, $M$ the molar mass of the fluid, $b$ the molar volume excluded by a fluid particle, $a$ a constant modelling the interactions between the fluid particles, and $c_{V}$ the specific heat capacity at constant volume.

The governing equations have been non-dimensionalized by introducing scaling quantities. The scales for mass density, temperature, and pressure are the 
corresponding quantities at the critical point:

$$
\rho_{c}=M /(3 b) \quad T_{c}=8 a /(27 R b) \quad P_{c}=a /\left(27 b^{2}\right)
$$

The velocity and time scales are the average sound speed in the system and the corresponding characteristic time:

$$
u_{c}=\sqrt{P_{c} / \rho_{c}} \quad t_{c}=L / u_{c}
$$

where $L$ is chosen to be the domain length.

These scaling quantities are reused in deriving the Reynolds, Weber, and Prandtl numbers, as well as the reduced heat capacity:

$$
R e=\rho_{c} u_{c} L / \mu \quad W e=u_{c}^{2} L^{2} /\left(\rho_{c} K\right) \quad \operatorname{Pr}=\mu c_{V} / \lambda \quad \tilde{c_{V}}=M c_{V} / R
$$

\subsection{Steady state approximation}

The governing equations support a steady state between two saturated phases. Its approximation close to the critical temperature was derived by Cahn and Hilliard [9]. The reduced mass density profile resulting from this approximation is given by

$$
\tilde{\rho}(x)=\frac{\tilde{\rho}_{1}+\tilde{\rho}_{\mathrm{v}}}{2}+\frac{\tilde{\rho}_{1}-\tilde{\rho}_{\mathrm{v}}}{2} \tanh \left(\frac{2 \tilde{x}}{\tilde{L}_{\mathrm{i}}}\right)
$$

where $\tilde{x}$ is the spatial coordinate, locating the interface at zero and the vapor and liquid phases at $-\infty$ and $+\infty$, respectively. The reduced mass densities of the saturated vapor and liquid phases are denoted by $\tilde{\rho}_{\mathrm{v}}$ and $\tilde{\rho}_{\mathrm{l}}$, and $\tilde{L}_{\mathrm{i}}$ is the reduced interface length at the same temperature. The last three quantities completely characterize the steady state and can be interpolated as functions of the reduced temperature:

$$
\left\{\begin{array}{l}
\tilde{\rho}_{\mathrm{v}}(\tilde{T})=1.00-1.86 \sqrt{1-\tilde{T}} \\
\tilde{\rho}_{\mathrm{l}}(\tilde{T})=1.00+2.08 \sqrt{1-\tilde{T}} \\
\tilde{L}_{\mathrm{i}}(\tilde{T})=\frac{2}{\sqrt{W e}}(-0.19+1.65 / \sqrt{1-\tilde{T}})
\end{array}\right.
$$

For the rest of the paper, the non-dimensional form is used but the tilde will be dropped for better readability.

\subsection{Space and time discretization}

The governing equations are discretized on a uniform Cartesian grid with size $\Delta x$. The space and time discretization methods were used by Pecenko et al. [6]. The approach was designed by Cockburn and Gau [10], by relying on a Runge-Kutta scheme developed by Shu and Osher [11]. 
The grid size is chosen to ensure that the interface is captured with at least ten grid points. The maximum time step, $\Delta t$, has to satisfy:

$$
\left\{\begin{aligned}
\left(\frac{\left|u_{0}\right| \pm c_{0}}{2 \Delta x}+\frac{\sqrt{\rho_{0}}}{\sqrt{4 W e \Delta x^{4}}}\right) \Delta t & \leq 1.5 \Gamma \\
\frac{4 \Delta t}{\operatorname{RePr} \Delta x^{2} \rho_{0}} & \leq 2.5 \Gamma \\
\frac{2 \Delta t}{\operatorname{Re} \Delta x^{2} \rho_{0}} & \leq 2.5 \Gamma
\end{aligned}\right.
$$

where $u_{0}$ and $\rho_{0}$ are the reduced velocity and mass density of the point whose equilibrium is studied, and $c_{0}$ is the reduced speed of sound evaluated at the same point. The empirical constant, $\Gamma$, is a safety factor to remain inside the stability region of the Runge-Kutta scheme. It has to be lower than one.

\section{Adaptation of the 1-D characteristic boundary conditions}

One-dimensional characteristics have been widely used in literature to specify open boundary conditions in computational aerodynamics. In the following, its extension to the DIM is presented in 1-D.

\subsection{Identifying the non-hyperbolic region for the Diffuse Interface Model}

Similar to the Navier-Stokes equations, the speeds of the waves supported by the DIM governing equations are

$$
\{u, u-\sqrt{\beta}, u+\sqrt{\beta}\} \text { with } \beta=2\left(\frac{P}{\rho}+\frac{4 T\left(3+c_{V}(-3+2 \rho)\right)}{c_{v}(-3+\rho)^{2}}\right)
$$

The speed of two of the waves becomes complex when $\beta$ is negative. This property undermines the application of the conventional 1-D characteristic boundary conditions. The corresponding regions are identified in grey on the $\rho$ $T$ diagram in Figure 1. It lies inside the multi-phase domain for a finite $c_{V}$ and fits perfectly on top of the transition curves as $c_{V} \rightarrow \infty$ (isothermal limit). Consequently, the 1-D characteristic boundary conditions can only be applied outside the multi-phase domain. When the interface moves closer to the boundary points, a different approach is discussed below.

\subsection{Designing a buffer layer absorbing the non-hyperbolic region}

We will explain the extension of the 1-D characteristic boundary conditions by recalling the simple example of a fluid experiencing phase transition from vapor to liquid and convected by a constant velocity field. Figure 2 depicts the different steps. The upper graphs present the mass density profiles, and the lower pictures indicate the position of the detectors. The role of these detectors will be explained in the following paragraphs.

The simulation mass density profile is indicated by the continuous line. The corresponding reference case run on a much larger domain is depicted in dashed 


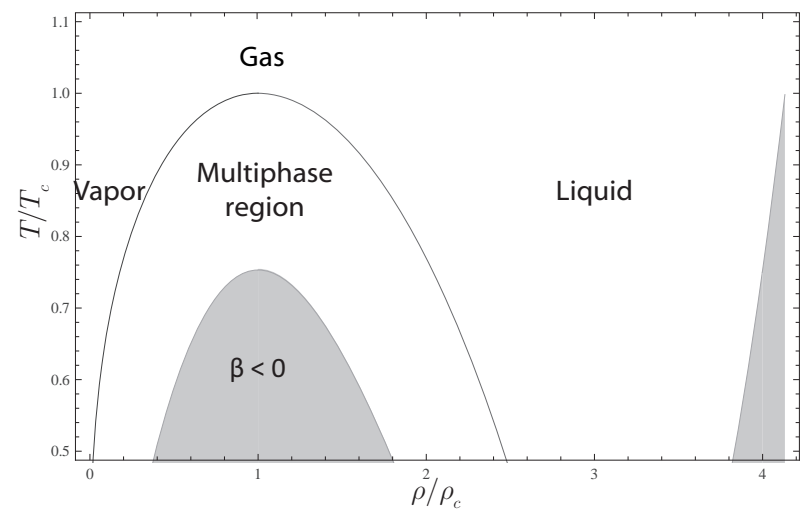

Figure 1: DIM non-hyperbolic region (in grey) for $c_{V}=3.05$ (water).

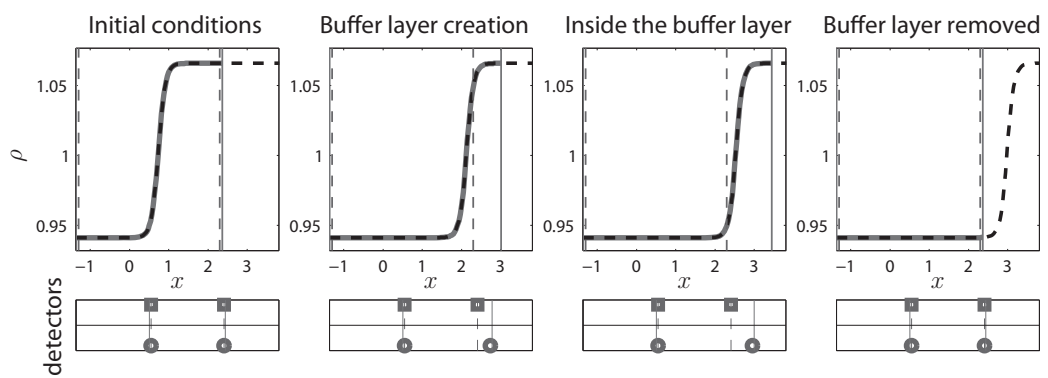

Figure 2: Application of the modified boundary conditions to the DIM.

black for the comparison on $D_{\mathrm{I}}$. The latter is delimited by the vertical dashed lines while $D_{\mathrm{C}}$ is indicated with vertical continuous lines. These two types of boundaries are not placed on top of each other at the beginning as boundary points are required for the spatial discretization scheme. These boundary points are initially located in the bulk phases where the governing equations exhibit hyperbolic properties. Therefore, the 1-D characteristic boundary conditions can be applied in these regions.

As the simulation runs, the velocity field, initialized as constant, convects the interface to the right. When the interface tries to cross the boundary, the equilibrium inside the elliptic region has to be maintained to prevent it from influencing the interior solution. This equilibrium is ensured by enlarging $D_{\mathrm{C}}$. The buffer layer creates a smooth transition between the single-phase boundary conditions (conventional 1-D characteristic) and the multi-phase region at the edge of $D_{\mathrm{I}}$. 
Based on the method of characteristics, the expression of the additional points is given by

$$
\frac{\partial \boldsymbol{U}}{\partial t}+\boldsymbol{A} \frac{\partial \boldsymbol{U}}{\partial x}=0 \Rightarrow \boldsymbol{U}_{x, t+d t}=\boldsymbol{R}\left(\begin{array}{c}
\boldsymbol{L}_{1} \boldsymbol{U}_{x-\lambda_{1} d t, t} \\
\boldsymbol{L}_{2} \boldsymbol{U}_{x-\lambda_{2} d t, t} \\
\boldsymbol{L}_{3} \boldsymbol{U}_{x-\lambda_{3} d t, t}
\end{array}\right)
$$

where $\boldsymbol{L}$ and $\boldsymbol{R}$ are the left and right eigenvectors of the matrix $\boldsymbol{A}$ while $\lambda_{i}$ are the eigenvalues. The matrix $\boldsymbol{A}$ is defined by retaining only the transport terms in the DIM governing equations. The eigenquantities are evaluated at the boundaries of $D_{\mathrm{I}}$. If the location corresponding to $\left(x-\lambda_{i} d t\right)$ is inside $D_{\mathrm{C}}$, the vector of conservative variables, $\boldsymbol{U}$, is interpolated. Otherwise, $\boldsymbol{U}_{x-\lambda_{i} d t, t}$ is calculated using the boundary conditions imposed in the far field.

The creation of the buffer region is triggered by increasing detectors. They are depicted by circles on the lower graphs. As they should prevent the multi-phase region from reaching the boundaries of $D_{\mathrm{C}}$, they are always located upstream of its edges: their position is updated as the buffer region is extended. The detection of the approaching multi-phase region is performed by computing the temperature and checking if the mass density lies within the range $\left[\rho_{\mathrm{v}}+\epsilon, \rho_{\mathrm{l}}-\epsilon\right]$. The saturated vapor and liquid densities are computed using equation (9) and $\epsilon$ is equal to $0.01\left(\rho_{\mathrm{l}}-\rho_{\mathrm{v}}\right)$.

As the simulation runs, the interface continues to enter the growing buffer region. However, the domain cannot be extended infinitely. The removal of the buffer layer has to be addressed while minimizing the interactions between the interface and $D_{\mathrm{I}}$. Deleting detectors are always located at the edges of the latter. They are depicted by squares on the lower graphs. When the density lies outside the range $\left[\rho_{\mathrm{v}}+\epsilon, \rho_{\mathrm{l}}-\epsilon\right]$, the DIM governing equations exhibit hyperbolic properties at the boundaries of $D_{\mathrm{I}}$ and the deleting detectors trigger the removal of the buffer layer.

\section{Numerical example}

The initialization procedure for the test case is first discussed. The performance of the extended boundary conditions on this test case is then evaluated. The main sources of perturbation induced by the extended boundary conditions are identified for the specific case $T=0.95$ and $u=0.1$. The analysis will be extended to the range of temperatures $T \in\{0.95,0.99,0.995,0.999\}$.

\subsection{Description of the test case}

\subsubsection{The reference case}

The waves supported by the governing equations have well-defined propagation speeds: $\left\{M_{\mathrm{f}}, M_{\mathrm{f}}-1, M_{\mathrm{f}}+1\right\}$ where $M_{\mathrm{f}}$ is the mean flow Mach number. For a specific simulation duration, the domain size of the reference case can be determined to prevent the travelling waves from bouncing back into $D_{\text {I }}$ before 
the end of the simulation, $t_{\mathrm{d}}$. Denoting by $L_{D_{\mathrm{I}}}$ the size of $D_{\mathrm{I}}$, the domain size of the reference case, $L_{\text {ref }}$, should satisfy:

$$
L_{\text {ref }} \geq L_{D_{\mathrm{I}}}+\left(\left|M_{\mathrm{f}}\right|+1\right) t_{\mathrm{d}}
$$

\subsubsection{The initial conditions}

The initial conditions model an interface between vapor and liquid travelling at a constant velocity. The temperature field is originally set to a constant. The corresponding saturated mass densities and interface length are computed using equation (9). The initial density profile is then computed using equation (8). The interface is located at the center of the domain, $x=0.5$.

When the temperature comes close to its critical value, the interface length increases. In order to prevent boundary effects from the interface in the beginning of the simulation, the size of the domain of interest is increased proportionally to the interface length:

$$
L_{D_{\mathrm{I}}}=10 L_{\mathrm{i}}(T)
$$

\subsubsection{The total duration and the error computation}

The total duration of the simulation is determined to allow the interface to leave $D_{\mathrm{C}}$. The distance separating the boundary of $D_{\mathrm{I}}$ from the final position of the interface is chosen as two times the width of the interface. From this distance and the initial velocity field, the total duration is deduced.

The relative error is computed by

$$
\operatorname{error}(t)=\max _{x \in D_{\mathrm{I}}}\left[\frac{\left|\rho(x, t)-\rho_{\mathrm{ref}}(x, t)\right|}{\rho_{\mathrm{ref}}(x, t)}\right]
$$

where $\rho$ and $\rho_{\text {ref }}$ are the reduced mass densities computed by the simulation and the reference case.

\subsection{Performances of the conventional and extended boundary conditions}

For the test cases, the reduced heat capacity of water is chosen: $\tilde{c_{V}}=3.05$. As depicted on Figure 1, this value allows the use of the conventional 1-D characteristic boundary conditions in the multi-phase region but only for a limited range of temperatures, including $T \in\{0.95,0.99,0.995,0.999\}$.

Table 1 depicts the maximum error reached in $D_{\text {I }}$ during the simulation. For the conventional boundary conditions, the interface crossing the boundary is treated as a simple wave. This approach creates reflected waves perturbing the interior solution. As for the extended boundary conditions, the sources of errors are identified in the next part.

\subsection{The error main characteristics}

The error induced by the extended boundary conditions shows a characteristic evolution in time. Figure 3 depicts the maximum error inside $D_{\text {I }}$ (continuous black 
Table 1: Conventional and extended boundary conditions comparison.

\begin{tabular}{|c|c|c|}
\hline Temperature & Error conventional b.c. & Error extended b.c. \\
\hline 0.95 & $6.5 \times 10^{-1}$ & $7.4 \times 10^{-3}$ \\
0.99 & $4.7 \times 10^{-2}$ & $1.8 \times 10^{-4}$ \\
0.995 & $1.2 \times 10^{-2}$ & $3.5 \times 10^{-5}$ \\
0.999 & $1.4 \times 10^{-3}$ & $1.0 \times 10^{-5}$ \\
\hline
\end{tabular}

line) and the $x$-coordinate where this maximum is reached (light line with squares) for the simulation $T=0.95$ and $u=0.1$. Both quantities are plotted as functions of time. We identified six phases:

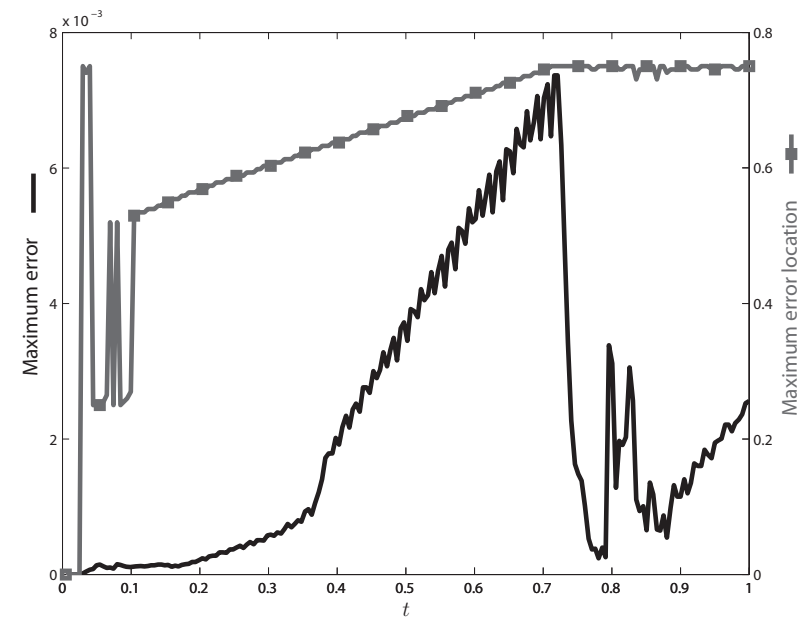

Figure 3: Error induced by the extended 1-D characteristic b.c.

- $0.025<t<0.05$ : away from the critical temperature, the initial mass density profile approximates the equilibrium between the two phases. Therefore, energy waves are created at the center and travel inside $D_{\mathrm{I}}$. As the interface is convected by a constant velocity field, the wave propagating with increasing $x$ reaches the right boundary before the one travelling with decreasing $x$ arrives at the left side. As the boundaries are not perfect, part of the wave is reflected. The error coming from the left side is larger than the one coming from the right side: two components of the characteristics are provided on the left while only one is supplied at the right boundary. 
- $0.05<t<0.1$ : the waves reflected at the boundaries influence the interior solution. As the interface equilibrium is very sensitive to perturbations, it is the major source of error with the left side.

- $0.1<t<0.375$ : the maximum error is located at the interface center.

- $0.375<t<0.71$ : the sudden increase of the error corresponds to the increase of the computational domain as the interface comes closer to the boundary. This extension requires information from the far field. The larger perturbation at the edge travels inside $D_{\text {I }}$ and modifies the equilibrium.

- $0.71<t<0.79$ : the sudden drop in the error at $t=0.71$ is caused by the interface leaving the domain of interest. The interface is still inside the computational domain but confined inside the buffer region. Its influence on the domain of interest is limited to heat conduction.

- $0.79<t<1$ : at $t=0.79$, the buffer region is removed. The perturbation originates from the conventional boundary conditions applied at the edge of the domain: they treat the parabolic effects caused by the heat dissipation terms as waves.

This analysis can be extended to $T \in\{0.95,0.99,0.995,0.999\}$. The error increases when the initial temperature moves away from the critical value as the initial mass density profile is a larger approximation of the equilibrium.

\section{Conclusion}

This paper focused on the extension of the 1-D characteristic open boundary conditions designed originally for the Navier-Stokes equations to the DIM in 1-D. Detectors are located at the boundaries and switch between the conventional single-phase boundary conditions and a multi-phase strategy.

Applications are made to the example of a fluid experiencing phase transition and convected by a constant velocity field. Compared to the use of the conventional boundary conditions where the $\rho-T$ diagram enables them, the additional multiphase strategy improves results. The test case at $T=0.999$ demonstrates that a fully developed interface can cross the boundaries with little effects. When the interface crossing the boundary is no longer fully developed, parabolic effects are the main sources of error and limit the applications. More research is needed for cases where these effects are dominant.

The approach developed can be extended to 2-D. Detectors are now placed along a closed loop path at the boundaries. When the increasing detectors watch an approaching bubble, the buffer region should adapt the computational domain with local 2-D extensions. The latter may complicate the implementation: the buffer region requires a dynamic data structure in 1-D to cut memory costs and the computation of the fluxes requires knowledge of the neighboring grid points. For the removal, the decision has to be made by a segment of deleting detectors. In the case of 3-D implementation, one can take advantage of the structure developed in 2-D by considering the 3-D computational field as a stack of 2-D fields. 


\section{Acknowledgement}

This research is supported by the Dutch Technology Foundation STW.

\section{References}

[1] Thompson, K., Time dependent boundary conditions for hyperbolic systems. J Comput Phys, 68(1), pp. 1-24, 1987.

[2] Hedstrom, G., Nonreflecting boundary conditions for nonlinear hyperbolic systems. J Comput Phys, 30(2), pp. 222-237, 1979.

[3] Poinsot, T. \& Lele, S., Boundary conditions for direct simulations of compressible viscous flows. J Comput Phys, 101(1), pp. 104-129, 1992.

[4] Gustafsson, B. \& Sundstrom, A., Incompletely parabolic problems in fluid dynamics. SIAM J Appl Math, 35(2), pp. 343-357, 1978.

[5] Sutherland, J. \& Kennedy, C., Improved boundary conditions for viscous, reacting, compressible flows. J Comput Phys, 191(2), pp. 502-524, 2003.

[6] Pecenko, A., van Deurzen, L., Kuerten, J. \& van der Geld, C., Non-isothermal two-phase flow with a diffuse-interface model. Int J Multiphase Flow, 37(2), pp. 149-165, 2011.

[7] Anderson, D. \& McFadden, G., A diffuse-interface description of internal waves in a near-critical fluid. Phys Fluids, 9(7), pp. 1870-1879, 1997.

[8] Dunn, J. \& Serrin, J., On the thermomechanics of interstitial working. Arch Rational Mech and Anal, 88, pp. 95-133, 1985.

[9] Cahn, J. \& Hilliard, J., Free energy of a nonuniform system. i. interfacial free energy. J Chem Phys, 28(2), pp. 258-267, 1958.

[10] Cockburn, B. \& Gau, H., A model numerical scheme for the propagation of phase transitions in solids. SIAM J Sci Comput, 17(5), pp. 1092-1121, 1996.

[11] Shu, C.W. \& Osher, S., Efficient implementation of essentially nonoscillatory shock-capturing schemes. J Comput Phys, 77(2), pp. 439-471, 1988. 